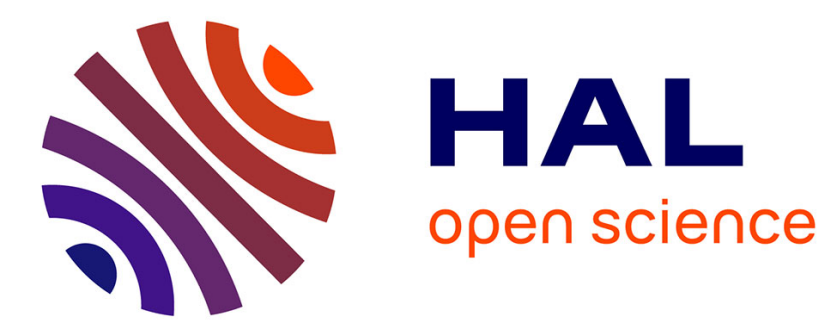

\title{
Robust reconstruction of the catalytic properties of thermal protection materials from sparse high-enthalpy facility experimental data
}

Francois Sanson, Francesco Panerai, Thierry E. Magin, Pietro Marco Congedo

\section{- To cite this version:}

Francois Sanson, Francesco Panerai, Thierry E. Magin, Pietro Marco Congedo. Robust reconstruction of the catalytic properties of thermal protection materials from sparse high-enthalpy facility experimental data. Experimental Thermal and Fluid Science, In press, 10.1016/j.expthermflusci.2018.03.028 . hal-01742595

\section{HAL Id: hal-01742595 \\ https://hal.inria.fr/hal-01742595}

Submitted on 4 Apr 2018

HAL is a multi-disciplinary open access archive for the deposit and dissemination of scientific research documents, whether they are published or not. The documents may come from teaching and research institutions in France or abroad, or from public or private research centers.
L'archive ouverte pluridisciplinaire HAL, est destinée au dépôt et à la diffusion de documents scientifiques de niveau recherche, publiés ou non, émanant des établissements d'enseignement et de recherche français ou étrangers, des laboratoires publics ou privés. 


\title{
Robust reconstruction of the catalytic properties of thermal protection materials from sparse high-enthalpy facility experimental data
}

\author{
Francois Sanson ${ }^{\mathrm{a}, *}$, Francesco Panerai ${ }^{\mathrm{b}}$, Thierry E. Magin ${ }^{\mathrm{b}}$, Pietro M. \\ Congedo $^{a}$ \\ ${ }^{a}$ INRIA Bordeaux Sud-Ouest, 200 Rue de la Vieille Tour, 33405 Talence, France \\ ${ }^{b}$ von Karman Institute for Fluid Dynamics, Chaussée de Waterloo 72, 1640 \\ Rhode-Saint-Genèse, Belgium
}

\begin{abstract}
Quantifying the catalytic properties of reusable thermal protection system materials is essential for the design of atmospheric entry vehicles. Their properties quantify the recombination of oxygen and nitrogen atoms into molecules, and allow for accurate computation of the heat flux to the spacecraft. Their rebuilding from ground test data, however, is not straightforward and subject to uncertainties. We propose a fully Bayesian approach to reconstruct the catalytic properties of ceramic matrix composites from sparse high-enthalpy facility experimental data with uncertainty estimates. The results are compared to those obtained by means of an alternative reconstruction procedure, where the experimental measurements are also treated as random variables but propagated through a deterministic solver. For the testing conditions presented in this work, the contribution to the measured heat flux of the molecular recombination is negligible. Therefore, the material catalytic property
\end{abstract}

\footnotetext{
*Corresponding author. Tel.: +33(0)5 24574112

Email address: francois.sanson@inria.fr (Francois Sanson)
} 
cannot be estimated precisely.Moreover, epistemic uncertainties are rigorously included, such as the unknown reference calorimeter catalytic property. Keywords: Uncertainty Quantification, Bayesian Inference, Catalysis, Thermal Protection Systems

\section{Introduction}

In the design of thermal protection systems for atmospheric entry vehicles, the catalytic properties of the heatshield material allow us to quantify the influence of the highly exothermic molecular recombinations occurring at the surface. In order to estimate these properties for a given material, groundbased high-enthalpy facilities are used to simulate flight conditions at the material surface and to provide relevant experimental data [1]. The plasma flow can be achieved using different techniques. In inductively-coupled plasma (ICP) wind tunnels, often referred to as plasmatrons, the plasma is generated by electromagnetic induction. A strong electromagnetic field ionizes the flow confined into a cylindrical torch and the plasma jet exits at subsonic speed into a low pressure test chamber that hosts material probes. The stagnation point conditions corresponding to a given spacecraft entry are reproduced for several minutes and the plasma flow carries sufficient energy to reproduce actual aerothermal loads experienced by a thermal protection system (TPS) in flight. Thanks to a flow of high chemical purity, plasmatron facilities are particularly suited to study gas/surface interaction phenomena for reusable TPS materials [2, 3, 4, 5, 6, 17, 8, 9, 10, 11] or composite ablative material [12]

. High-temperature experiments enable characterizing the catalytic properties of the tested TPS sample by combining direct measurements using various 
diagnostics and a numerical reconstruction based on computational fluid dynamics (CFD) simulations.

Even for well-characterized facilities, the determination of catalytic properties is affected by the noise present in the experimental data. The quantification of uncertainties in high-enthalpy experiments has previously been studied in the literature [13, 14, 15, 16]. In particular, in our previous work [16], we evaluated the uncertainties on catalytic properties by coupling a deterministic catalytic property estimation with a Polynomial Chaos (PC) expansion method. The probabilistic treatment of the uncertainties helped mitigating over-conservative uncertainty estimates found in the literature by computing confidence intervals. The influence of the epistemic uncertainty on the catalytic property of a reference calorimeter used in the reconstruction was also investigated in [16]. However, the method developed has two shortcomings: the number of experiments is limited and statistics about the measurements distribution are not available, even though they are an essential input for the PC approach.

Two important aspects are explored in the present work. First, we develop a robust methodology for quantifying the uncertainties on the catalytic property following a Bayesian approach. The Bayesian framework has already been successfully applied to the study of graphite nitridation [14] and hightemperature kinetics [17], for model parameter estimation as well as for experimental design [18, but it is a novel approach for the case of reusable materials, bringing a new insight on the ceramic matrix composites on which this paper focuses. In a Bayesian approach, one computes the probability distribution of the possible values of a quantity of interest compatible with 
the experimental results and with prior information about the system. This is fundamentally different from the PC approach proposed in [16]. While both approaches aim at quantifying the uncertainty on the catalytic properties, the experimental data are direct inputs of the deterministic solver combined to the PC method, whereas they are observed outputs of a model for the Bayesian method.

Second, a thorough comparison between the two methods is developed in order to explain the results obtained in view of their conceptual differences. We investigate the case of two experiments necessary for the reconstruction of the flow enthalpy and material catalytic property. The PC approach sequentially considers the experiments, whereas the Bayesian approach merges them into a unique simultaneous reconstruction. Additionally, the Bayesian approach has a major advantage: it allows us to determine the catalytic property of a reference copper calorimeter used in the reconstruction methodology, along with the catalytic property of the sample material. The robustness of the method is also examined for cases where the problem is not well posed, for instance when there are too many parameters to rebuild, and no sufficient information from the experiments.

In this contribution, we propose to revisit measurements performed in a high-enthalpy ICP wind-tunnel (Plasmatron) at the von Karman Institute for Fluid Dynamics (VKI) to characterize the catalytic response of ceramic matrix composites. Based on the robust uncertainty quantification methodology developed, we will assess whether accurate information on the catalytic properties of these thermal protection materials can be extracted from the experimental data. The paper is structured as follows. In section 2, we 
recall the main features of the combined experimental/numerical methodology developed at VKI to analyze data obtained in the Plasmatron facility, and then, present the sources of experimental uncertainties involved in the process. In section 3 , we reformulate the problem of determining the catalytic properties in a Bayesian framework. In Section 4, we apply this approach to experimental data presented in [4] and compare our results to the uncertainty estimate obtained in [16] by means of the PC approach.

\section{Experimental/numerical methodology}

The present study uses a set of data measured during an experimental campaign documented in [4]. The first section briefly recalls the quantities measured experimentally for each testing conditions and their associated uncertainties, whereas the next section introduces the numerical simulations performed to rebuild quantities that cannot be directly measured. The last section introduces some uncertainty quantification terminology.

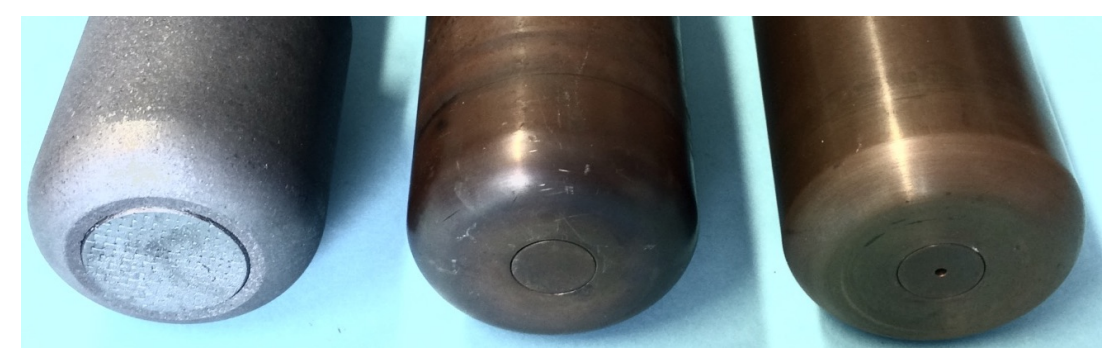

Figure 1: ESA standard probes ( $5 \mathrm{~cm}$ diameter) used for the measurements performed in the Plasmatron facility: (left to right) stagnation-point probe holding a material sample, copper calorimeter, and water-cooled Pitot probe. 


\subsection{Experimental setup}

In order to derive the catalytic property $\gamma$ of a ceramic matrix composite sample, the reconstruction methodology used in [4] is based on two sequential experiments. The first step consists in rebuilding the free stream enthalpy $h_{e}$ of the plasma flow, using the cold wall heat flux measurement $q_{c w}$ from a copper calorimeter (see Fig. 1) of catalytic property $\gamma_{\text {ref }}$. The uncertainties on the heat flux measurements were computed to be $\pm 10 \%$. Note that the quantity $\gamma_{\mathrm{ref}}$ is a source of large uncertainties [16]. A commonly adopted assumption is to consider the surface as fully catalytic [2, 19]. While this is a conservative practice, there is compelling evidence that the actual surface of copper calorimeters is not fully catalytic, owing to the rapid oxidation of copper upon exposure to plasma. Numerous studies have been dedicated to characterize the catalytic properties of copper and its surface oxides $\left(\mathrm{CuO}\right.$ and $\left.\mathrm{Cu}_{2} \mathrm{O}\right)$ [10, 13, 20, 21, 22, 23, 24, 25, 26, 27, 28, 29, 30, 31, 32, 33, 34, 35, 36, 37.

Together with the heat flux, the total pressure is measured during the first experiment. A water-cooled Pitot probe is introduced in the Plasmatron flow in order to measure the dynamic pressure $P_{d}$ (featuring an uncertainty of $\pm 6 \%$ ). The surface temperature of water-cooled probes $T_{\mathrm{cw}}$ is known by measuring the differential of temperature between the inlet and outlet water lines. The static pressure $P_{\mathrm{s}}$ of the test chamber is measured with a $2 \mathrm{~Pa}$ accuracy.

In a second step, hot wall measurements are performed on the TPS material sample in order to determine its catalytic property $\gamma$, for a known test condition determined through the rebuilding of cold-wall measurements. The emissivity $\varepsilon$ of the sample is measured with $10 \%$ accuracy. The front 


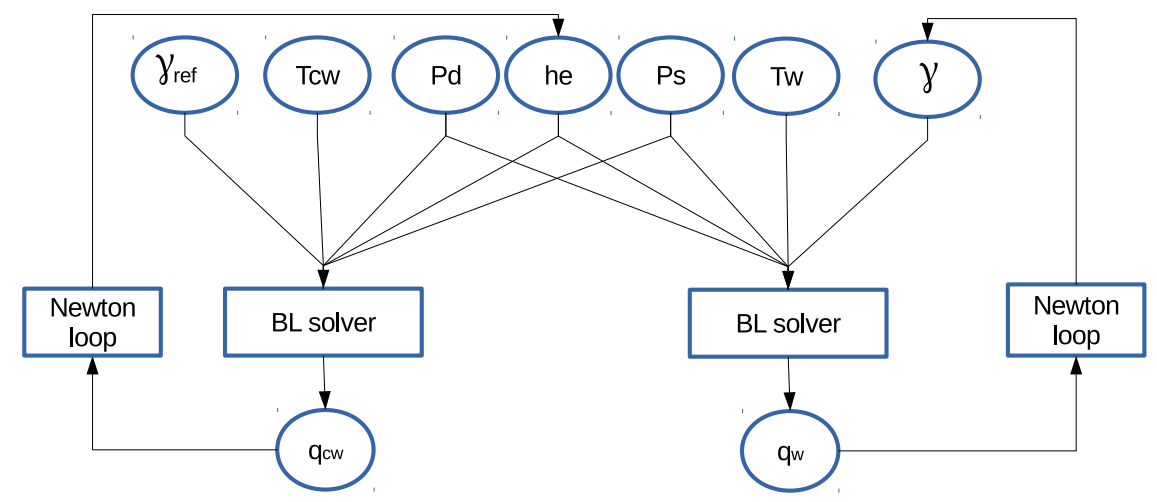

Figure 2: Flow chart of the deterministic estimation of material catalytic property

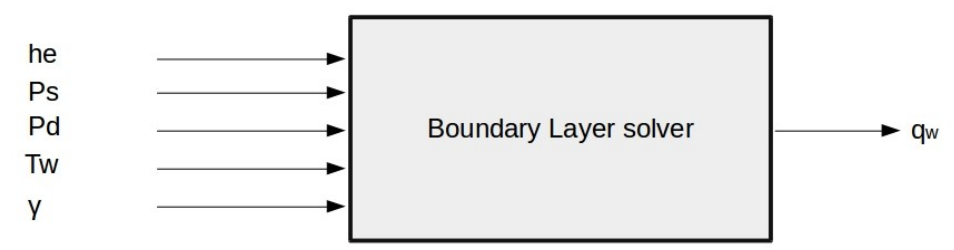

Figure 3: Flow chart of the BL solver with its main inputs

surface temperature of the sample $T_{\mathrm{w}}$ is also measured by means of a two-color pyrometer (affected by a $1 \%$ error). The heat flux $q_{w}$ radiated by the TPS is then computed using the Stefan-Boltzmann law.

It is further assumed that the free stream flow is identical during both experiments and that local thermodynamic equilibrium (LTE) holds at the edge of the boundary layer. At steady state, the surface radiated heat flux is assumed to be equal to the incoming heat flux from the plasma flow. 


\subsection{Numerical computations}

The Plasmatron flow conditions in front of the TPS test sample are rebuilt using experimental data and a 1D non-equilibrium Boundary Layer (BL) solver [38, 39] that propagates the flow field quantities from the outer edge of the BL to the stagnation point. The rebuilding methodology is sketched in Fig. 2. The BL solver computes the stagnation point heat flux $q_{c w}$ (or $q_{w}$ for the TPS sample) that, mathematically, is a function of the probe geometry, the surface temperature $T_{c w}$ (or $T_{w}$ for the TPS sample), and the wall catalytic property of the reference calorimeter $\gamma_{\text {ref }}$ (or $\gamma$ for the TPS sample), given the following set of the plasma flow free stream parameters: enthalpy $h_{e}$, pressure $p_{e}$, and velocity $v_{e}$. The PEGASE library [40], embedded with the boundary layer solver, provides the physico-chemical properties of the plasma flow.

The BL solver can be called by a rebuilding code using Newton's method to determine the quantities $h_{e}$ and $\gamma$ in a two-step strategy involving one rebuilding per experiment. The static pressure $p_{e}$ is assumed to be equal to the static pressure $P_{s}$ measured in the chamber. The enthalpy rebuilding uses the measured dynamic pressure $P_{d}$ to compute the free stream velocity $v_{e}$ using a viscous correction, as well as the heat flux $q_{c w}$ measured at the surface of the reference calorimeter to reconstruct the free stream enthalpy $h_{e}$. In a second step, the results from the second experiment and the flow field parameters computed during the first step are combined to determine the sample material catalytic property $\gamma$.

Despite the fact that a large number of inputs are measured or unknown, the method is fully deterministic and provides no indication about the outputs uncertainty. Our previous work [16] was based on the propagation of 
Table 1: Measured quantities used for the flow and sample material characterization

$\begin{array}{llc}\text { Symbol } & \text { Variable } & \text { Uncertainty } \\ P_{d, \text { meas }} & \text { Dynamic pressure } & 6 \% \\ P_{s, \text { meas }} & \text { Static pressure } & 0.3 \% \\ q_{c w, \text { meas }} & \text { Heat flux } & 10 \% \\ T_{c w, \text { meas }} & \text { Probe temperature } & 10 \% \\ T_{w, \text { meas }} & \text { TPS temperature } & 1 \% \\ \varepsilon & \text { Emissivity } & 5 \%\end{array}$

uncertainties using this inverse deterministic solver.

\subsection{Uncertainty characterization in catalytic property reconstruction}

The determination of the TPS catalytic property directly depends on experimental data, and intrinsically carries the uncertainty associated with actual measurements. Uncertainty Quantification (UQ) tools model and quantify the error associated to the variables computed using uncertain inputs. Table 1 reviews the measured quantities and their uncertain. The uncertainties can be classified into three categories:

- The measured quantities (MQ) come from the two experimental steps described earlier. The following quantities are measured: $T_{c w \text {,meas }}$, $q_{c w \text {,meas }}, T_{w \text {,meas }}, \varepsilon_{\text {meas }}, P_{d \text {,meas }}, P_{s, \text { meas }}$, namely the calorimeter probe temperature, the calorimeter probe heat flux, the sample temperature, the sample emissivity, the plasma jet dynamic pressure and static pressure. Note that the heat flux from the second experiment $\left(q_{w, \text { meas }}\right)$ is not directly measured but derived from quantities $T_{w \text {,meas }}$ and $\varepsilon_{\text {meas }}$ 
using Stefan-Boltzmann's law: $q_{w, \text { meas }}=\sigma \varepsilon_{\text {meas }} T_{w \text {,meas }}^{4}$. The MQ are aleatory quantities that are assumed to be noisy versions of their true values denoted $T_{c w}, q_{c w}, T_{w}, \varepsilon, P_{d}, P_{s}$. In this study, they are modeled as realization of a Gaussian distribution. The quantity $T_{c w \text {,meas }}$ denotes the measurement of the probe temperature, so we have:

$$
T_{c w, \text { meas }}=T_{c w}+\zeta
$$

where $\zeta$ is the realization of a zero mean Gaussian random variable.

- The quantities of interest (QoI) are the unknown quantities crucial to engineering applications. In this study, the sample and the probe catalytic properties denoted $\gamma$ and $\gamma_{\text {ref }}$, along with the flow enthalpy $h_{e}$, are the QoIs. The objective is not only to compute the most likely value of the catalytic property or the one that minimizes the square error but to compute the full probability distribution of all admissible values of the QoI given the measurements for a thorough quantification of uncertainties.

- The Nuisance Parameters (NP) are unknown quantities that must be estimated along with the $\mathrm{QoI}$ in order to estimate the sample catalytic property. Quantities $T_{c w}, T_{w}, P_{d}, P_{s}, \varepsilon$ are NPs as they have to be estimated in order to run the BL solver used to derive the sample catalytic property.

\section{Bayesian-based approach}

One objective of this work is to make a joint estimation of the catalytic properties $\gamma_{\text {ref }}$ and $\gamma$ of the reference calorimeter and sample material, re- 
spectively, along with the flow enthalpy $h_{e}$, for a given set of experiments. In [16], a polynomial chaos expansion was built on top of the inverse deterministic solver described earlier. In this section, we detail the derivation of the probability distribution of these quantities given the experimental results using a Bayesian approach. This probability distribution of these quantities is referred to as the posterior distribution. This distribution carries all the necessary information for the uncertainty quantification analysis. It provides a robust estimate of the uncertainty through confidence intervals and the variance.

In section 3.1, the posterior distribution is decomposed into a ratio of probabilities using Bayes' rule (Eq. 4) that can be numerically evaluated. Detailed calculations of each terms of the decomposition are then presented in section 5. Finally, the posterior distribution is numerically evaluated using a Markov Chain Monte Carlo (MCMC) algorithm described in appendix. Figure 4 summarizes the rebuilding methodology from a Bayesian perspective. Note that, contrary to the deterministic strategy illustrated in Figure 2, the QoI are rebuilt using both experiments simultaneously. The differences in the two approaches are further discussed in section 4.

\subsection{Bayesian framework}

We recall that the heat flux to the sample material wall $q_{w, \text { meas }}$ is completely defined by the material emissivity $\varepsilon_{\text {meas }}$ and temperature $T_{w \text {,meas }}$ through Stefan-Boltzmann's law. Introducing the vector of measured quantities $m=\left(T_{c w, \text { meas }}, q_{c w \text {,meas }}, T_{w, \text { meas }}, \varepsilon_{\text {meas }}, P_{d \text {,meas }}, P_{s, \text { meas }}\right)$, the posterior probability is then indicated as follows:

$$
P\left(\gamma_{\mathrm{ref}}, h_{e}, \gamma \mid m\right) \text {. }
$$




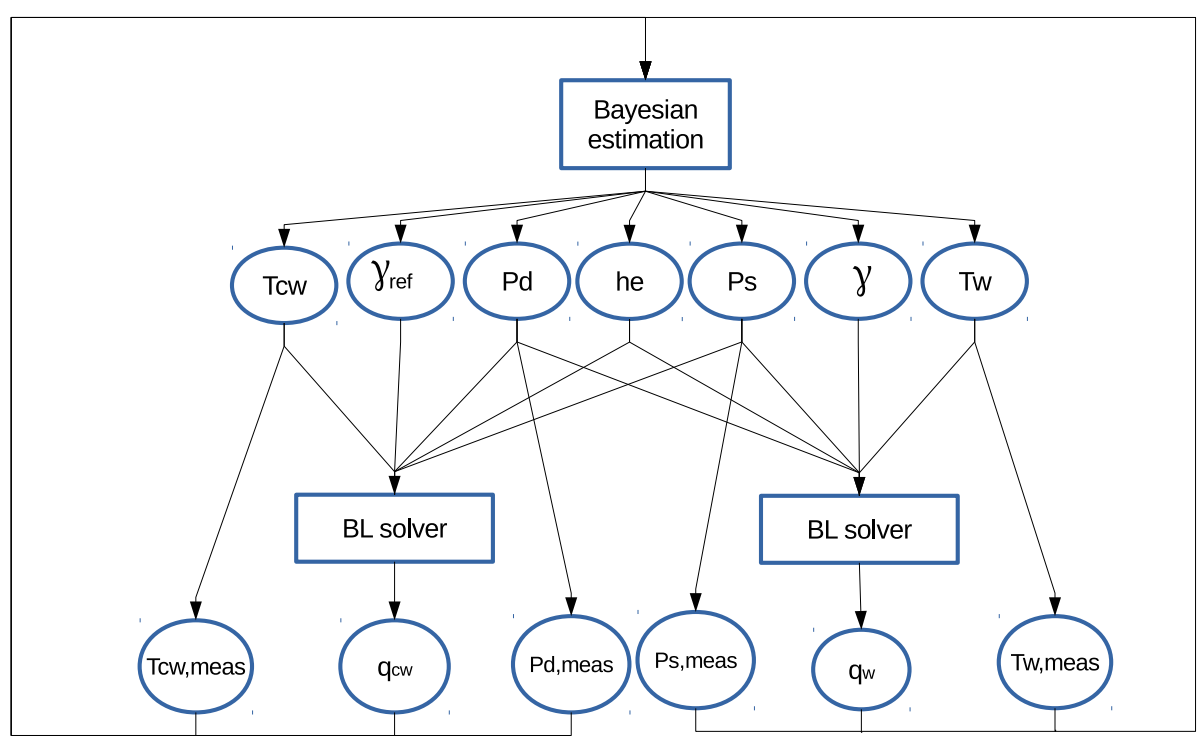

Figure 4: Flowchart of the Bayesian-based estimation of material catalytic properties.

Furthermore, the vector of NP is introduced as $\omega_{\text {nuis }}=\left(T_{c w}, T_{w}, \varepsilon, P_{d}, P_{s}\right)$. The posterior distribution (Eq.2) can alternatively be computed by integrating the so-called non-marginalized posterior $P\left(\gamma_{\text {ref }}, h_{e}, \gamma, \omega_{n u i s} \mid m\right)$, over all the $\mathrm{NP}$

$$
P\left(\gamma_{\mathrm{ref}}, h_{e}, \gamma, \mid m\right)=\int_{\omega_{\text {nuis }}} P\left(\gamma_{\mathrm{ref}}, h_{e}, \gamma, \omega_{\text {nuis }} \mid m\right) d \omega_{\text {nuis }}
$$

Let us now focus on the non-marginalized posterior from Eq. 3. The flowchart in Fig. 4 shows the relationships between the unknowns $\gamma_{\text {ref }}, h_{e}, \gamma$, $\omega_{\text {nuis }}$ and the MQ (i.e., the vector $m$ ) and how they interact with each other. In order to evaluate Eq. 3, Bayes' rule is applied as follows:

$$
P\left(\gamma_{\mathrm{ref}}, h_{e}, \gamma, \omega_{\text {nuis }} \mid m\right)=\frac{P\left(m \mid \gamma_{\mathrm{ref}}, h_{e}, \gamma, \omega_{\text {nuis }}\right) P\left(\gamma_{\mathrm{ref}}, h_{e}, \gamma, \omega_{\text {nuis }}\right)}{P(m)}
$$

where $P\left(m \mid \gamma_{\mathrm{ref}}, h_{e}, \gamma, \omega_{\text {nuis }}\right)$ is the likelihood, $P\left(\gamma_{\mathrm{ref}}, h_{e}, \gamma, \omega_{\text {nuis }}\right)$ the prior, and $P(m)$ a normalization factor such that the probabilities add up to 
one. The likelihood quantifies the amount of information carried by the measurements to the QoI and the NP. It is the probability of observing the measured quantities knowing the QoI and the NP. It measures the compatibility between the measurements and the value of unknown parameters, such as the catalytic property of the material sample. When the value of the catalytic property is compatible with the experimental results, the likelihood increases. The amount of this increase is directly related to the amount of information brought by the measurements. If the measurements are very informative, the increase (or decrease if the catalytic property gets less and less compatible with the experiments) is very steep. The prior accounts for the knowledge of the unknown parameters before any experiment. In our case, as scarce prior information is available for $\omega_{\text {nuis }}$ and $h_{e}$, uniform priors are considered. As $\gamma$ and $\gamma_{\text {ref }}$ are defined on the interval [0;1], a beta distribution with parameters $\alpha=1$ and $\beta=1$ is chosen with a support of $\left[10^{-8} ; 1\right]$. The next section is devoted to the determination of the likelihood.

\section{Results}

This section illustrates the results derived from the application of the Bayesian framework to the problem of interest. The objective is twofold: i) to compute an estimate of the QoI (flow enthalpy $h_{e}$ and catalytic properties $\gamma_{\text {ref }}$ and $\gamma$ of the reference calorimeter and sample material) and ii) to compare the results with the uncertainty estimates obtained in [16] from a more standard PCE approach. In order to demonstrate the potential of the Bayesian approach, two sets of experimental conditions are selected among the experiments presented in [4]. They are denoted as S1 and S8, as detailed 
in Table 2, For both experiments, we study the following two cases:

a. The calorimeter reference probe catalytic property $\gamma_{\text {ref }}$ is assumed to be constant and equal to 0.1 (Section 4.1). The results for the posterior distribution are presented. Uncertainty estimates are compared with the ones obtained in [16]. Qualitative and quantitative explanations of the differences between the results obtained by the two approaches are given.

b. Secondly, the probe catalytic property is treated as an unknown quantity determined along with the other NPs and QoIs (section 4.3). Again the results are compared against the method developed in [16].

\subsection{Constant calorimeter reference probe catalytic property}

Quantity $\gamma_{\text {ref }}$ is assumed to be constant and equal to 0.1 , focusing on the computation of the posterior distribution of the flow enthalpy $h_{e}$ and material catalytic property $\gamma$. The statistical moment and the $95 \%$ confidence interval are given in Tables 4 and 3 for quantities $h_{e}$ and $\gamma$, respectively. Their mean values are in good agreement with the nominal results obtained in [4]. Figure 5 shows their distributions for sample S1. It is observed that the reconstructed quantities $h_{e}$ and $\gamma$ both have symmetrical distributions. Theses results can be related to the typical S-shape enthalpy versus catalytic property curve reported in the literature [16, 36]. In this case, most of the posterior lies within the high gradient zone on the S-shape, meaning that the small changes in catalytic property induce large variations in the computed heat flux at the wall as they are related through a one to one mapping in that region. In other words, if the measured heat flux takes values in that 
region, it is expected that the catalytic property posterior will have limited variance. The Maximum A Posteriori (MAP) is defined as the maximum of the posterior density probability. It is an alternative point estimator to the mean of a QoI. In the special case of a Gaussian posterior, the MAP and the mean are equals. The analysis of sample S8 yields similar results and conclusions. The relative error computed as the ratio of the mean and the 95\% confidence interval (CI) is one order of magnitude large for the catalytic property compared to the rebuilt enthalpy.

\subsection{Comparison with Polynomial-Chaos approach}

This section compares the proposed Bayesian approach to the PC approach presented in [16]. In the deterministic solver, the two steps of the experiments are taken sequentially (cf. Figure 2 in [16]): first the flow field is computed using the results from the first experiment, namely measurements of cold-wall heat flux, as well as the static and dynamic pressures. Then, the sample catalytic property is determined using the quantities rebuilt from the first experiment. In order to propagate uncertainties, a polynomial approximation of the solver was derived and used to generate the statistical moments of the sample catalytic property. More precisely, the MQ are the only inputs to the

$\begin{array}{ccccccccc}\text { Sample } & q_{c w, \text { meas }} & P_{s} & P_{d} & T_{c w, \text { meas }} & h_{e} & T_{w, \text { meas }} & \varepsilon_{\text {meas }} & \gamma \\ & {\left[\mathrm{kW} . \mathrm{m}^{-2}\right]} & {[\mathrm{Pa}]} & {[\mathrm{Pa}]} & {[\mathrm{K}]} & {\left[\mathrm{MJ.kg}^{-1}\right]} & {[\mathrm{K}]} & {[-]} & {[-]} \\ \text { S1 } & 195 & 1300 & 75 & 350 & 6.0 & 1200 & 0.78 & 7.3 \mathrm{e}-3 \\ \text { S8 } & 410 & 3000 & 64 & 350 & 9.7 & 1400 & 0.88 & 3.0 \mathrm{e}-3\end{array}$

Table 2: Deterministic conditions for material samples S1 and S8. Here, reported values of $h_{e}$ and $\gamma$ are determined using the standard rebuilding procedure detailed in [4]. 

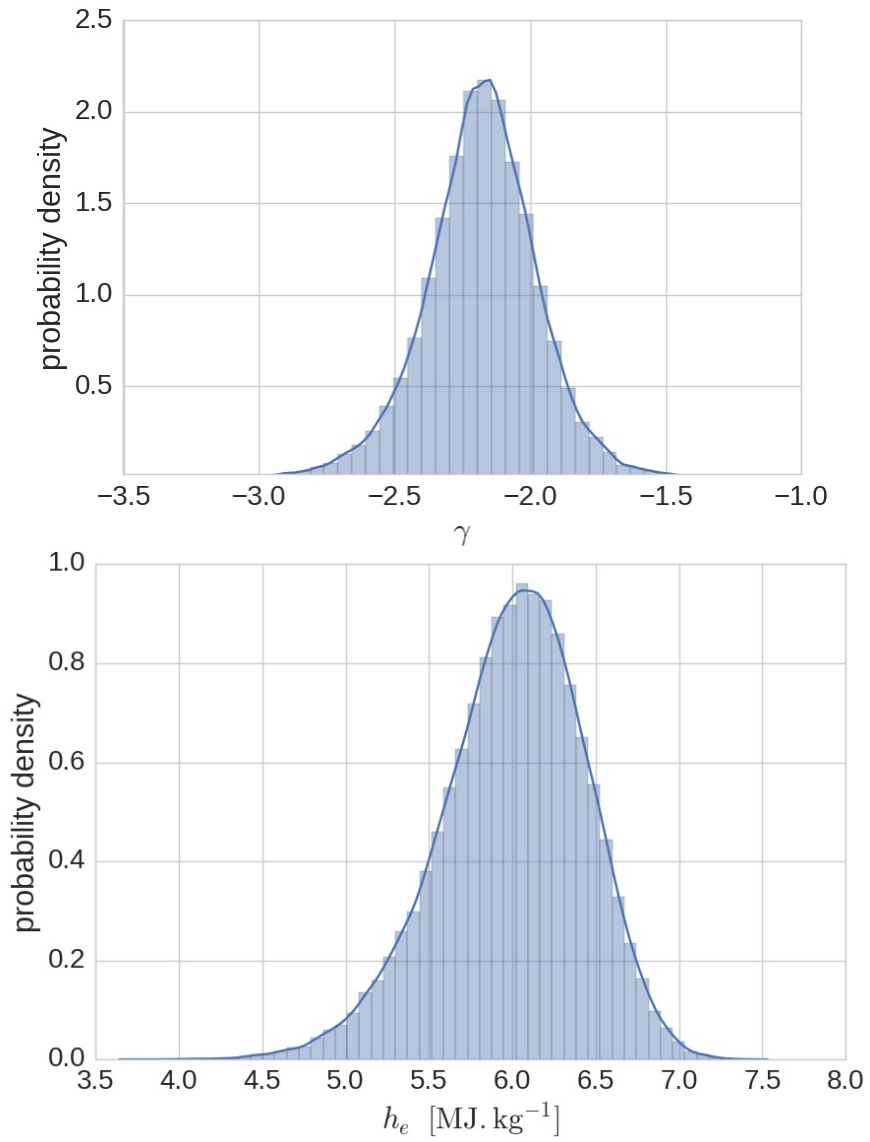

Figure 5: Bayesian reconstruction $\left(\gamma_{\mathrm{ref}}=0.1\right)$ of the material catalytic property on a semi-log scale (top) and flow enthalpy on a linear scale (bottom) for material sample S1. 


$\begin{array}{cccccc}\text { Sample } & \text { Mean } & \text { SD } & \text { MAP } & 95 \% \text { CI } & \text { UQ }(95 \% \text { CI })[\%] \\ \text { S1 } & 6.0 & 0.43 & 6.06 & {[5.06 ; 6.76]} & 28.3 \\ \text { S8 } & 9.7 & 0.43 & 6.66 & {[8.80 ; 10.51]} & 17.6\end{array}$

Table 3: Flow enthalpy $h_{e}\left[\mathrm{MJ} \mathrm{kg}^{-1}\right]$ statistics obtained by means of the Bayesian approach $\left(\gamma_{\mathrm{ref}}=0.1\right)$

$\begin{array}{cccccc}\text { Sample } & \text { Mean } & \text { SD } & \text { MAP } & 95 \% \text { CI } & \text { UQ(95\% CI })[\%] \\ \text { S1 } & 7.4 \mathrm{e}-3 & 4.1 \mathrm{e}-03 & 6.2 \mathrm{e}-3 & {[2.4 \mathrm{e}-3 ; 1.7 \mathrm{e}-2]} & 197.2 \\ \text { S8 } & 3.7 \mathrm{e}-3 & 1.8 \mathrm{e}-03 & 2.7 \mathrm{e}-3 & {[1.4 \mathrm{e}-3 ; 8.38 \mathrm{e}-3]} & 188.6\end{array}$

Table 4: Material catalytic property $\gamma$ statistics obtained by means of the Bayesian approach $\left(\gamma_{\mathrm{ref}}=0.1\right)$

polynomial approximation in [16], whereas the probe catalytic property $\gamma_{\text {ref }}$ is kept constant. In order to include the uncertainty of the probe catalytic property, several polynomial approximations of the solver are computed in [16] for different values of $\gamma_{\text {ref }}$.

In the following section, we highlight the differences between the PC and Bayesian methods. Both qualitative (section 4.2.1) and quantitative (section 4.2.2 illustrations are provided. Sample S1 conditions are chosen for this exercise.

\subsubsection{Qualitative differences between the PC and Bayesian methods}

The PC and Bayesian approaches tackle the problem from different angles leading to different results. The main differences between the two methods can be summarized as follows. 
- The experimental data accumulated during the two reconstructions are not exploited in the same way. In the Bayesian formulation, the measurements are treated silmutaneously in order to reconstruct the catalytic property distribution at once (cf. Fig. 4), whereas the PC approach coupled with the deterministic inverse problem use sequential reconstructions of each quantity (see Fig. 2). In particular, the flow enthalpy is estimated in the PC approach only using the first experiment, whereas the Bayesian approach uses information from both experiments to rebuild the flow enthalpy. As mentioned in [41], in [4, 16] the link between the two experiments acts like a valve: the information (or uncertainty) only goes one way. The information from the second experiment does not flow back to the determination of the flow enthalpy. Only information from the first experiment goes to the second reconstruction via the boundary layer edge enthalpy $h_{e}$. This method presents some similarities with the Cut-model used in Bayesian networks [41, but it generally leads to a wrong posterior distribution.

- Input uncertainties are modeled differently. The PC approach makes stronger hypothesis about the input distribution by assuming that its mean is the experimental value. In the Bayesian framework, it is only assumed that the experimental value obtained is sampled from a Gaussian distribution with mean function of the NP and QoI. This is a strong assumption since a single experimental result can be significantly different from the mean value.

- Not only the input measurements are not modeled the same way, but 
the way they are propagated is also different. The PC approach, and the results presented in [16], depend on the deterministic method used to solve the inverse problem. In fact, the PC approach only provides the variance of the outputs and higher statistical moments. On the other hand, the Bayesian method leads to an unbiased asymptotically efficient estimation of the sample catalytic property [42, 43].

- Finally, the Bayesian approach offers more flexibility in order to add uncertainties without major issues in the computational time, whereas the PC approach is limited by the problem of the curse of dimensionality [44, 45], i.e., the lack of convergence speed of the numerical method when an increasing number of uncertainties is considered. Moreover, the Bayesian framework is well-suited for modeling epistemic uncertainty, such as the reference probe catalytic property. In the method developed in [16], this property is not modeled as a distribution, since no information is available to characterize it. Therefore, a limited set of values of $\gamma_{\text {ref }}$ on an arbitrary interval are tested to provide an envelope of the uncertainty on the QoI. On the other hand, the Bayesian implementation can use the information collected during the experiments to compute a posterior distribution of the reference probe catalytic property. Using that posterior distribution, the method yields a much more precise estimation of the uncertainty in the QoI along with an estimation of $\gamma_{\text {ref }}$. 


\subsubsection{Quantitative differences between the PC and Bayesian methods}

In this section, numerical tests are performed with sample S1 (see Table 2). The comparison focuses on the distributions of the material catalytic properties, as well as on the modeling uncertainties coming from the unknown catalytic property $\gamma_{\text {ref }}$ of the reference calorimeter.

The reconstructions of the material catalytic property $\gamma$ are first compared using a constant value of $\gamma_{\text {ref }}$ equal to 0.1. Although this case may be unrealistic, since the probe catalytic property is rarely well known, it illustrates the differences between the two methods in a basic setting. Figure 6 shows differences in the sample catalytic property distribution obtained with the PC [16] and Bayesian methods. Note that, in Table 5, the first moment of the two distributions are very close, however the standard deviations and the confidence intervals are significantly larger for the distribution obtained with the Bayesian approach. This explains the much larger magnitude of the relative error. Moreover, the MAP estimates are substantially different: for the Bayesian case, the distribution is skewed and the most probable value and the mean values of the sample catalytic property are different. This is not observed when using the Polynomial Chaos, since the catalytic property distribution is Gaussian.

Since $\gamma_{\text {ref }}$ is rarely known, its variability and influence on the QoI are also investigated here. In particular, the approach used in [16] for including the epistemic uncertainty due to $\gamma_{\text {ref }}$ is compared to the Bayesian implementation. For the PC method, the uncertainty on the QoI due to the MQs are computed for discrete values of $\gamma_{\mathrm{ref}}$, whereas for the Bayesian method, $\gamma_{\mathrm{ref}}$ is a priori 


$\begin{array}{cccccc}\text { Method } & \text { Mean } & \text { SD } & \text { MAP } & 95 \% \text { CI } & \text { UQ }(95 \% \text { CI })[\%] \\ \text { Polynomial Chaos } & 0.00747 & 1.6 \mathrm{e}-03 & 0.007 & {[0.0045 ; 0.0094]} & 65.6 \\ \text { Bayesian } & 0.00747 & 4.1 \mathrm{e}-03 & 0.0059 & {[0.0024 ; 0.017]} & 195.4\end{array}$

Table 5: Comparison between the statistics of catalytic property $\gamma$ for material sample S1 obtained by means of the PC and Bayesian approaches $\left(\gamma_{\mathrm{ref}}=0.1\right)$.

unknown. In Figure 7, the cumulative density function (CDF) of the flow enthalpy derived from the PC approach is plotted for the extreme values of $\gamma_{\text {ref }}$, i.e. 1 and 0.01 , as well as derived from the Bayesian approach with an a priori unknown value of $\gamma_{\text {ref }}$. For both values of $\gamma_{\text {ref }}$, the CDF obtained by means of the PC approach exhibits a much steeper increase compared to the state-of-the-art Bayesian approach, leading to a much more precise estimate of the uncertainty on the enthalpy. This is due to the different degree of knowledge of the probe catalytic property for the two methods. Since the Bayesian implementation uses the measurements to estimate the probe catalytic property, the uncertainty due to the epistemic quantity decreases. Conversely, for the PC implementation, no information about the probe catalytic property is available, leading to an overestimation of the uncertainty in the enthalpy. In summary, the Bayesian method makes a better use of the information available from the experiments and provides an optimal, reliable estimate of the uncertainty. The distributions of the material catalytic property obtained by means of the Bayesian approach with $\gamma_{\mathrm{ref}}$ a priori unknown will be studied in the following section. 


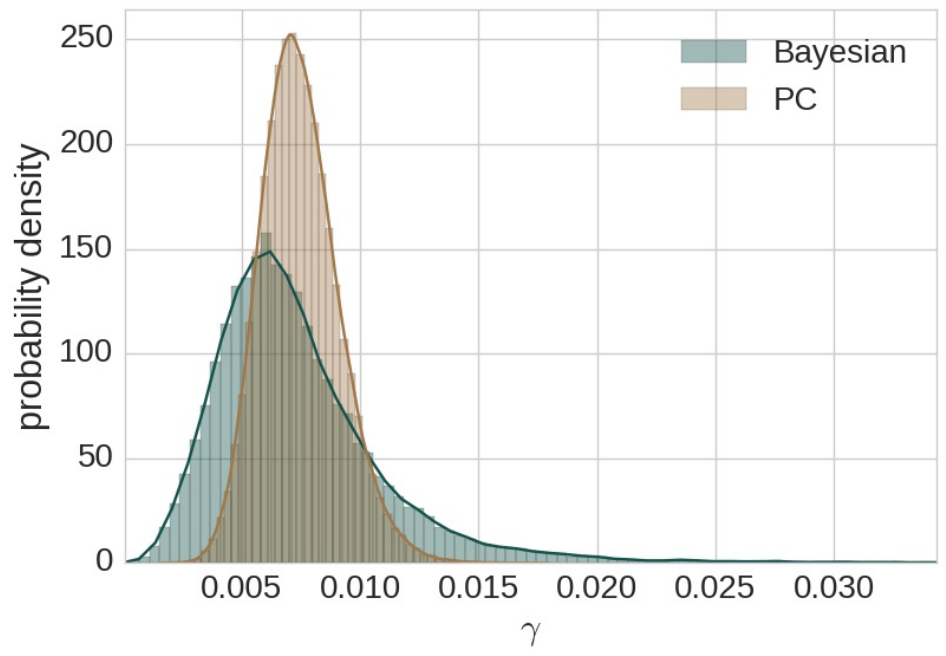

Figure 6: Comparison between the distributions of the material catalytic property for sample $\mathrm{S} 1$ obtained by means of the PC and Bayesian approaches $\left(\gamma_{\mathrm{ref}}=0.1\right)$.

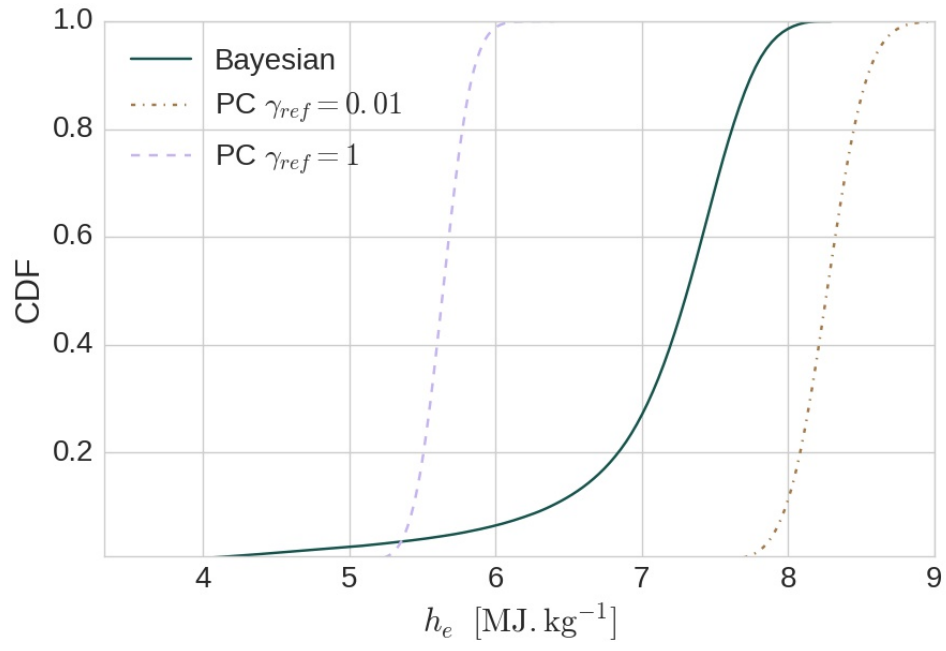

Figure 7: Comparison between the CDF of the flow enthalpy obtained by means of the PC $\operatorname{approach}\left(\gamma_{\mathrm{ref}}=0.01\right.$ and 1$)$ and Bayesian $\left(\gamma_{\mathrm{ref}}\right.$ a priori unknown $)$ approach. 


\subsection{Case where the reference probe catalytic property is unknown}

In contrast to an approach commonly followed in the literature, we consider here the value of the probe catalytic property to be unknown, instead of arbitrarily set to a constant value. Therefore, $\gamma_{\text {ref }}$ is determined along with the other unknown quantities and the target distribution is the new posterior: $P\left(\gamma_{\text {ref }}, h_{e}, \gamma, \omega_{\text {nuis }} \mid T_{c w \text {,meas }}, q_{c w \text {,meas }}, T_{w \text {,meas }}, \varepsilon_{\text {meas }}, P_{\mathrm{d} \text {,meas }}, P_{\mathrm{s} \text {,meas }}\right)$. Hence, the influence of the probe catalytic property on the sample catalytic property uncertainty can be rigorously quantified. Due to the increase in the number of unknowns and in order to increase the speed of convergence of the MCMC algorithm, the Markov Chain is adapted using the Adapted Metropolis (AM) algorithm presented in [46] with a modification from [47] (see algorithms 4 and 5). This approach is more precise and more flexible than the approach used in [16] where a robust brute force method is presented to explore the influence of the probe catalytic property. In this work, the Bayesian approach gives finer results thanks to a better knowledge of the probe catalytic property.

Results obtained for the estimation of the material catalytic property $\gamma$, flow enthalpy $h_{e}$, and reference calorimeter catalytic property $\gamma_{\text {ref }}$ for the two samples S1 ans S8 are presented. Figure 8 shows the distribution of $\gamma_{\text {ref }}$ and Table 6 summarizes their statistics. Means and variances results should be used with care as the computed distributions are extremely far from Gaussian. Based on the experimental data of sample S1, the computed value for the reference probe catalytic property is 0.018 , as shown in table 6. This result indicates that the assumption of $\gamma_{\mathrm{ref}}=0.1$ utilized in [4] is over-conservative. The results obtained for $\gamma_{\text {ref }}$ for the two conditions (S1 and S8) are rather different but not contradictory. The relative error 
is extremely large. Note that with sample $\mathrm{S} 1, \gamma_{\text {ref }}$ can be estimated with slightly more accuracy than with sample S8. This observation shows that the precision of the determination of the estimation of $\gamma_{\text {ref }}$ depends on the experimental conditions and not only on the accuracy on the measurements. The addition of an extra NP increases the uncertainty on the QoI and other NP. Figure 9 shows the distribution of $h_{e}$ for sample S1 that can be compared to earlier results presented in Figure 5 for the case with a constant $\gamma_{\text {ref }}$. The distribution support is significantly increased and shifted toward higher values. This change can be explained by a simple physical reasoning: for the same value of the experimental heat flux measurement, the reference probe catalytic property has been estimated by means of the Bayesian approach to a value of 0.018 much lower than 0.1 . Consequently, the contribution to the heat flux due to catalytic recombination is lower than in the $\gamma_{\text {ref }}=0.1$ case and the contribution from the convective heat flux therefore becomes larger and the flow enthalpy is estimated as well to a higher value than in the $\gamma_{\text {ref }}=0.1$ case. Figure 10 shows the distribution of the material catalytic property for samples S1 and S8. For both samples, the material catalytic property uncertainty is much more widespread with respect to the previous case where quantity $\gamma_{\text {ref }}$ was assumed to be constant. In particular, the support of the distribution covers eight orders of magnitudes and does not present a clear maximum for a precise a posteriori estimation. In the case of an unknown quantity $\gamma_{\text {ref }}$, the experiments do not contain sufficient information. Indeed, one can notice that the posterior distribution is similar to the beta prior distribution, meaning that the likelihood is not informative in this case. The reason for this loss of information is investigated in the following paragraph. Nevertheless, 


$\begin{array}{cccccc}\text { Sample } & \text { Mean } & \text { Standard deviation } & \text { MAP } & 95 \% \text { Confidence interval } & \text { UQ }(95 \% \text { CI })[\%] \\ \text { S1 } & 0.042 & 0.095 & 0.018 & {[0.0075 ; 0.29]} & 672.6 \\ \text { S8 } & 0.091 & 0.17 & 0.0009 & {[1.3 \mathrm{e}-07 ; 0.71]} & 780.2\end{array}$

Table 6: Reference probe catalytic property $\gamma_{\text {ref }}$ statistics obtained by means of the Bayesian approach.

even though the support of the distribution is extremely large and seems non informative, some remarks can be made about the CDF. Figure 11 compares the CDF of $\gamma$ based on the sample S1 in the two cases where $\gamma_{\text {ref }}$ is either constant (equal to 0.1 ) or unknown. The constant $\gamma_{\text {ref }}$ case is actually a worst case scenario that overestimates the molecular recombination rate at the surface of the sample. The unknown $\gamma_{\text {ref }}$ case shows that the actual material sample catalytic property is certainly lower. Its distribution is hardly usable as it is, especially for the low values of $\gamma$ since for those the posterior is very similar to the arbitrary prior chosen for this study. However, the CDF remains useful to estimate probabilities and confidence intervals.

Now, we investigate reasons for the large increase in the $\gamma$ uncertainty for an unknown $\gamma_{\text {ref }}$ quantity compared to the constant case. It is partially due to the addition of $\gamma_{\text {ref }}$ as NP but also to the lower estimation of $\gamma_{\text {ref }}=0.018$ leading to an increase in the estimated flow enthalpy. The dependence of material catalytic property versus the flow enthalpy is weak. By inspecting the distributions of $\gamma$ in Figure 10, one notices that these are flat in particular for sample S8. In other words, the sample catalytic property does not influence the measured heat flux for the tested conditions. It follows that scarce information from the measured heat flux can be used to estimate $\gamma$. 


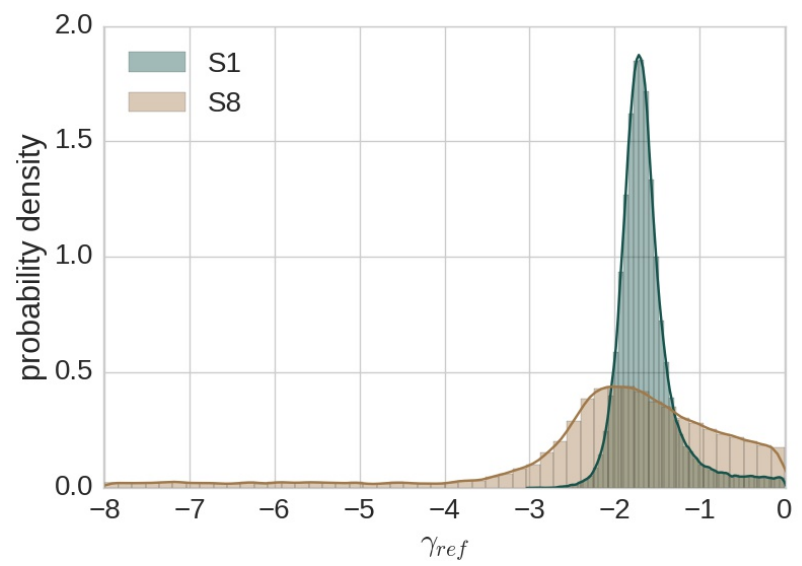

Figure 8: Distribution of the reference probe catalytic property $\gamma_{\mathrm{ref}}$ for samples S1 and S8 on a semi-log scale, obtained by means of the Bayesian approach.

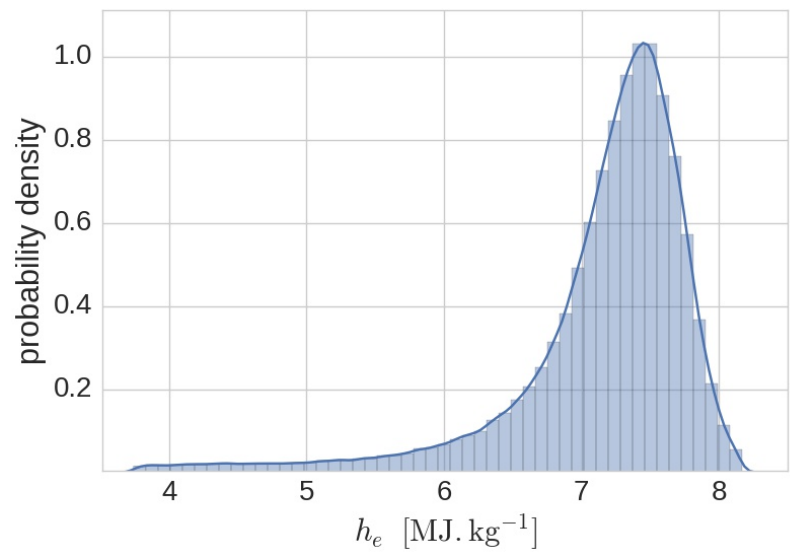

Figure 9: Distribution of the flow enthalpy $h_{e}$ for sample S1 obtained by means of the Bayesian approach ( $\gamma_{\text {ref }}$ a priori unknown). 


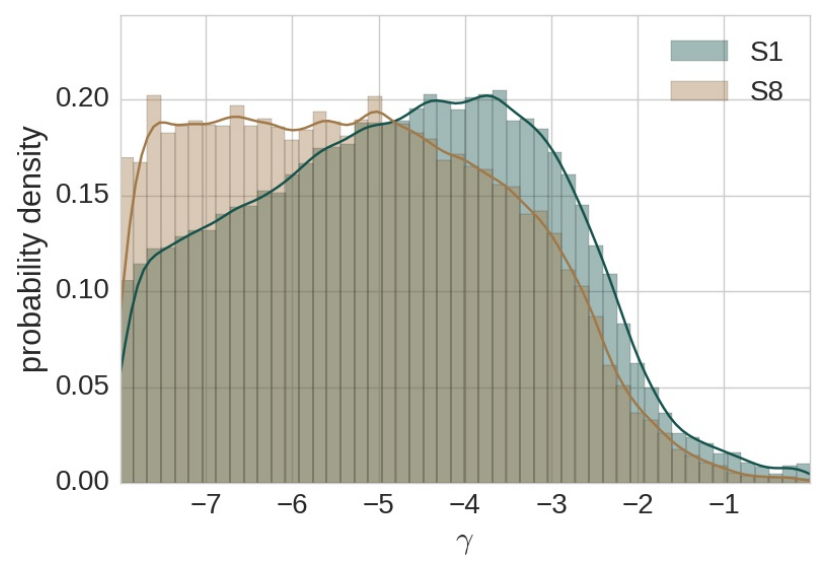

Figure 10: Distribution of the material catalytic property $\gamma$ for samples S1 and S8 on a semi-log scale, obtained by means of the Bayesian approach ( $\gamma_{\mathrm{ref}}$ a priori unknown).

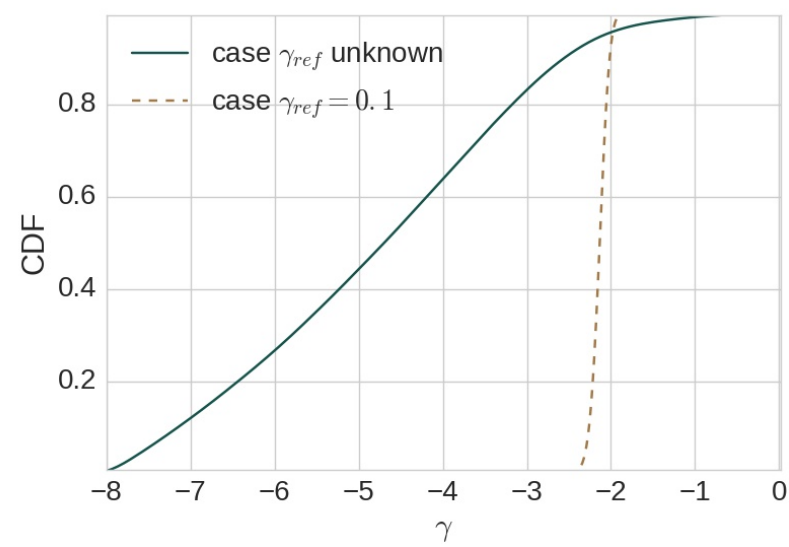

Figure 11: CDF of the material catalytic property $\gamma$ for sample S1 on semi-log scale, obtained by means of the Bayesian approach $\left(\gamma_{\mathrm{ref}}=0.1\right.$ and $\gamma_{\mathrm{ref}}$ a priori unknown). 
In particular even the slight uncertainty on the determination of the flow enthalpy is associated with large uncertainty on the catalytic property of the material. This means that for the range of enthalpy between $4 \mathrm{MJ} / \mathrm{kg}$ and 8 $\mathrm{MJ} / \mathrm{kg}$ (see Fig. 9 ) it is challenging to precisely estimate the sample catalytic property for those testing conditions. To illustrate the problem, Figure 12 shows the Bayesian reconstruction of the sample catalytic property for a case where the probe catalytic property $\gamma_{\text {ref }}$ is set to a constant value of 0.02 . The sample material experiment considered here is S1. The bivariate distribution of the flow enthalpy $h_{e}$ and material catalytic property $\gamma$ show that, for a given flow enthalpy, the curve of enthalpy versus catalytic property has a very low gradient. Even though the probe catalytic property is known and constant, the uncertainty is comparable to the case where the probe catalytic property has to be computed. Therefore the increase of uncertainty in the sample catalytic property is due to the experimental conditions rather than to the precision of the measurements. This remark shows that while the specific experimental condition had been selected based on a relevant flight environment, it is not optimal for accurately estimating the TPS material catalytic property. A similar conclusion can be made for sample S8. 


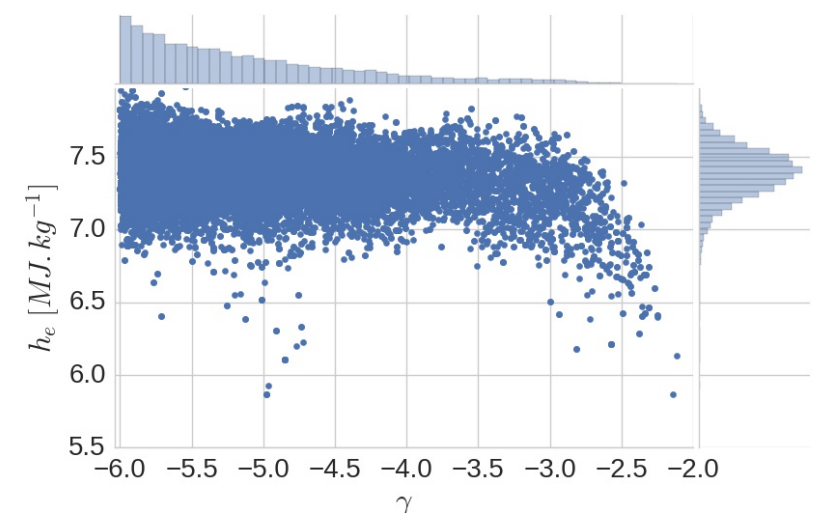

Figure 12: Bivariate distribution of the flow enthalpy $h_{e}$ and material catalytic property $\gamma$ for sample S1 on semi-log scale obtained by means of the Bayesian approach $\left(\gamma_{\text {ref }}=0.02\right)$.

\section{Conclusion}

In this study, a rigorous method for estimating the catalytic property of a material and the associated uncertainties is presented. By comparing a Bayesian approach with an alternative uncertainty quantification method presented in [16], we showed that the two methods do not yield the same results. By construction, the Bayesian approach is more adapted to cases where a limited number of experiments are available while the approach presented in [16] makes stronger assumptions on the measurement distribution that are only valid when a large number of experiments is available. Moreover, we found that the Bayesian approach is also more flexible as it can naturally include epistemic variables such as the unknown reference calorimeter catalytic property uncertainty.

The uncertainty analysis carried out in the case of the unknown reference calorimeter catalytic property showed that the experimental set up is not adequate to precisely estimate the catalytic property of a given material. 
For the testing conditions presented in this work, the contribution to the measured heat flux of the molecular recombination is negligible. Therefore, the material catalytic property cannot be estimated precisely. Conversely, in this study, we were able to have some estimation of the reference calorimeter catalytic property. We have found that the assumption of constant value $\gamma_{\text {ref }}=0.1$ is wrong and introduces a bias in the estimation of the material catalytic property.

As future work, we propose to identify experimental conditions that are optimal for accurately estimating the TPS material catalytic properties.

\section{Acknowledgment}

The authors would like to thanks Andrien Todeschini for the fruitful discussions and remarks on the Bayesian approach.

\section{Appendix A: Determination of the likelihood}

The likelihood represents the link between the MQ, the QoI and NP and is directly related to the experiments. The two experiments from the first and second steps are independent, so that the likelihood can be rewritten as:

$$
\begin{aligned}
P\left(m \mid \gamma_{\mathrm{ref}}, h_{e}, \gamma, \omega_{\text {nuis }}\right)= & P\left(T_{c w, \text { meas }}, q_{c w \text {,meas }}, P_{d, \text { meas }}, P_{s, \text { meas }} \mid \gamma_{\text {ref }}, h_{e}, \omega_{\text {nuis }}\right) \\
& \times P\left(T_{w, \text { meas }}, \varepsilon_{\text {meas }} \mid \gamma, h_{e}, \omega_{\text {nuis }}\right)
\end{aligned}
$$

where $P\left(T_{c w \text {,meas }}, q_{c w \text {,meas }}, P_{d \text {,meas }}, P_{s, \text { meas }} \mid \gamma_{\text {ref }}, h_{e}, \omega_{\text {nuis }}\right)$ is the likelihood of the measurements obtained during the enthalpy reconstruction and $P\left(T_{w \text {,meas }}\right.$, 
$\left.\varepsilon_{\text {meas }} \mid \gamma, h_{e}, \omega_{\text {nuis }}\right)$ is the likelihood of the measurements obtained during the catalytic property reconstruction. Note that quantity $\gamma_{\text {ref }}$ is solely involved in the first experiment, whereas quantity $\gamma$ in the second one. However, both experiments are still connected through the free stream conditions (such as the enthalpy $h_{e}$ ) that are assumed to be constant for both probes that are injected sequentially in the plasma jet. The two likelihoods can still be computed in two different steps, as shown in the following sections.

\subsection{Derivation of the first experiment likelihood}

The enthalpy rebuilding step does not involve $\varepsilon_{\text {meas }}$ and $T_{w, m e a s}$. The expression becomes:

$$
\begin{aligned}
& P\left(T_{c w, \text { meas }}, q_{c w, \text { meas }}, P_{d, \text { meas }}, P_{s, \text { meas }} \mid \gamma_{\text {ref }}, h_{e}, \omega_{\text {nuis }}\right)=P\left(T_{c w, \text { meas }} \mid \gamma_{\text {ref }}, h_{e}, \omega_{\text {nuis }}\right) \\
& \times P\left(q_{c w, \text { meas }} \mid \gamma_{\mathrm{ref}}, h_{e}, \omega_{\text {nuis }}\right) P\left(P_{d, \text { meas }} \mid \gamma_{\mathrm{ref}}, h_{e}, \omega_{\text {nuis }}\right) \\
& \times P\left(P_{s, \text { meas }} \mid \gamma_{\mathrm{ref}}, h_{e}, \omega_{\text {nuis }}\right),
\end{aligned}
$$

since the measurements are considered independent.

Each term from the right hand side of Eq.11 has to be evaluated individually. For instance, for the cold wall surface temperature, one has that:

$$
\begin{aligned}
P\left(T_{c w, \text { meas }} \mid \gamma_{\mathrm{ref}}, h_{e}, \omega_{\text {nuis }}\right) & =P\left(T_{c w, \text { meas }}=T_{c w}+\zeta \mid \gamma_{\mathrm{ref}}, h_{e}, \omega_{\text {nuis }}\right) \\
& =\frac{1}{\sqrt{2 \pi \sigma_{T_{c w, \text { meas }}}^{2}}} \exp \left(-\frac{\left(T_{c w, \text { meas }}-T_{c w}\right)^{2}}{2 \sigma_{T_{c w, \text { meas }}}^{2}}\right) .
\end{aligned}
$$

The last equality comes from the fact that $\zeta$ is a zero mean Gaussian random 
variable. Very similarly, one has

$$
\begin{aligned}
P\left(q_{c w, \text { meas }} \mid \gamma_{\mathrm{ref}}, h_{e}, \omega_{\text {nuis }}\right) & =P\left(q_{c w, \text { meas }}=q_{c w}+\zeta \mid \gamma_{\mathrm{ref}}, h_{e}, \omega_{\text {nuis }}\right) \\
& =\frac{1}{\sqrt{2 \pi \sigma_{q_{c w} \text { meas }}^{2}}} \exp \left(-\frac{\left(q_{c w, \text { meas }}-q_{\mathrm{cw}}\right)^{2}}{2 \sigma_{q_{c w}, \text { meas }}^{2}}\right), \\
P\left(P_{d, \text { meas }} \mid \gamma_{\mathrm{ref}}, h_{e}, \omega_{\text {nuis }}\right) & =P\left(P_{d, \text { meas }}=P_{d}+\zeta \mid \gamma_{\mathrm{ref}}, h_{e}, \omega_{\text {nuis }}\right) \\
& =\frac{1}{\sqrt{2 \pi \sigma_{P_{d, \text { meas }}}^{2}}} \exp \left(-\frac{\left(P_{d, \text { meas }}-P_{d}\right)^{2}}{2 \sigma_{P_{d, \text { meas }}}^{2}}\right), \\
P\left(P_{s, \text { meas }} \mid \gamma_{\mathrm{ref}}, h_{e}, \omega_{\text {nuis }}\right) & =P\left(P_{s, \text { meas }}=P_{s}+\zeta \mid \gamma_{\mathrm{ref}}, h_{e}, \omega_{\text {nuis }}\right) \\
& =\frac{1}{\sqrt{2 \pi \sigma_{P_{s, \text { meas }}}^{2}}} \exp \left(-\frac{\left(P_{s, \text { meas }}-P_{s}\right)^{2}}{2 \sigma_{P_{s, \text { meas }}}^{2}}\right) .
\end{aligned}
$$

Note that $q_{\mathrm{cw}}$ can be computed using the BL solver as it is a function of $\gamma_{\text {ref }}, h_{e}$, and $\omega_{\text {nuis. }}$. Finally Eq. 6 becomes:

$$
\begin{aligned}
& P\left(T_{c w, \text { meas }}, q_{c w, \text { meas }}, P_{d, \text { meas }}, P_{s, \text { meas }} \mid \gamma_{\mathrm{ref}}, h_{e}, \omega_{\text {nuis }}\right)= \\
& \frac{1}{(2 \pi)^{2} \sigma_{P_{s, \text { meas }}} \sigma_{P_{d, \text { meas }}} \sigma_{T_{c w, \text { meas }}} \sigma_{q_{c w, \text { meas }}}} \exp \left(-\frac{\left(P_{s, \text { meas }}-P_{s}\right)^{2}}{2 \sigma_{p_{s}}^{2}}\right) \\
& \times \exp \left(-\frac{\left(P_{d, \text { meas }}-P_{d}\right)^{2}}{2 \sigma_{P_{d, \text { meas }}}^{2}}-\frac{\left(T_{c w, \text { meas }}-T_{c w}\right)^{2}}{2 \sigma_{T_{c w, \text { meas }}}^{2}}-\frac{\left(q_{\mathrm{cw}, \text { meas }}-q_{\mathrm{cw}}\right)^{2}}{2 \sigma_{q_{c w, \text { meas }}}^{2}}\right) .
\end{aligned}
$$

\subsection{Derivation of the second experiment likelihood}

For the second set of experiments the material sample is tested in order to measure its catalytic property $\gamma$. The catalytic property rebuilding step consists in computing $P\left(T_{w \text {,meas }}, \varepsilon_{\text {meas }} \mid \gamma, h_{e}, \omega_{\text {nuis }}\right)$. In the rebuilding procedure, the heat flux radiated by the TPS is assumed to be equal to the heatflux 
$q_{\mathrm{w}}$ from the flow to the TPS, which is computed by means of the BL solver. Mathematically we have:

$$
q_{w}\left(\gamma, h_{e}, \omega_{n u i s}\right)=\sigma \varepsilon T_{w}^{4}
$$

Following the same procedure as for the enthalpy rebuilding, the likelihood for the catalytic property rebuilding has the following form:

$$
P\left(T_{w, \text { meas }}, \varepsilon_{\text {meas }} \mid \gamma, h_{e}, \omega_{\text {nuis }}\right)=P\left(T_{w, \text { meas }} \mid \gamma, h_{e}, \omega_{\text {nuis }}\right) P\left(\varepsilon_{\text {meas }} \mid \gamma, h_{e}, \omega_{\text {nuis }}\right)
$$

and the following expression can be computed:

$$
P\left(T_{w, \text { meas }} \mid \gamma, h_{e}, \omega_{n u i s}\right)=\frac{1}{\sqrt{2 \pi \sigma_{T_{w, \text { meas }}}^{2}}} \exp \left(-\frac{\left(T_{w, \text { meas }}-T_{w}\right)^{2}}{2 \sigma_{T_{w, \text { meas }}}^{2}}\right) .
$$

Similarly, it follows that:

$$
P\left(\varepsilon_{\text {meas }} \mid \gamma, h_{e}, \omega_{\text {nuis }}\right)=\frac{1}{\sqrt{2 \pi \sigma_{\varepsilon_{\text {meas }}}^{2}}} \exp \left(-\frac{\left(\varepsilon_{\text {meas }}-\frac{q_{w}}{\sigma T_{w}^{4}}\right)^{2}}{2 \sigma_{\varepsilon_{\text {meas }}^{2}}^{2}}\right) .
$$

Therefore, the catalytic property likelihood becomes:

$$
\begin{aligned}
P\left(T_{w, \text { meas }}, \varepsilon_{\text {meas }} \mid \gamma, h_{e}, \omega_{\text {nuis }}\right)= & \frac{1}{\sqrt{2 \pi \sigma_{T_{w, \text { meas }}}^{2}}} \exp \left(-\frac{\left(T_{w, \text { meas }}-T_{w}\right)^{2}}{2 \sigma_{T_{w, \text { meas }}}^{2}}\right) \\
& \frac{1}{\sqrt{2 \pi \sigma_{\varepsilon_{\text {meas }}}^{2}}} \exp \left(-\frac{\left(\varepsilon_{\text {meas }}-\frac{q_{w}}{\sigma T_{w}^{4}}\right)^{2}}{2 \sigma_{\varepsilon_{\text {meas }}}^{2}}\right) .
\end{aligned}
$$

Injecting Eqs. 12 and 17 in Eq. 5 provides an explicit way to numerically evaluate the likelihood. Unfortunately, even though there are analytical solutions for the likelihood and the prior distribution, in order to compute the posterior, it is necessary to compute the normalization factor in Eq. 4. In this study, this is computationally intractable. To bypass that issue, a classical 
Markov Chain Monte Carlo method is used to directly compute the posterior without having to evaluate the normalization factor. In fact, the Metropolis algorithm enables to sample from the posterior distribution [42, 48, 49, 50]. Details of the implementation are given in appendix B.

\section{Appendix B: Monte Carlo Markov Chain and Metropolis Algo- rithm}

The Metropolis algorithm builds a Markov Chain which is asymptotically distributed as the posterior distribution $\gamma \mid m$. It is an acceptance-rejection method where the acceptance criteria assures the convergence of the Markov Chain toward the desired distribution [50]. Complete proof of the convergence of the Algorithm can be found in [42, 48]. In this section, the basics of the algorithm and the specificity of the implementation are presented.

Consider a random walk Markov Chain $X_{n}$ on state space $\mathcal{S}$. In the case studied, $\mathcal{S}$ contains all the admissible values of the NPs and QoIs. Consider two states $\mathrm{x}$ and $\mathrm{y} \in \mathcal{S}$, the probability to go from $\mathrm{x}$ to y is $\mathrm{P}(\mathrm{x}, \mathrm{y})$ referred as the transition probability. Let $\pi(x)$ be the distribution of $X_{n}$, if $\sum_{x \in \mathcal{S}} \pi(x) P(x, y)=\pi(y)$. Then, the distribution $\pi$ is said to be invariant or stationary. In the special case of random walks, the invariant distribution is unique and the random walk converges to $\pi$ asymptotically (see [49] or [48]). In other words, no matter where the Markov Chain started, we have,

$$
\lim _{n \longrightarrow \infty} X_{n} \sim \pi
$$

The Metropolis algorithm uses the right transition probability $P(x, y)$ such as $\pi$ is the distribution of interest (the QoI distribution). It uses this results 
from Markov Chain theory cf. [42] or [49] for more further details) :

If $\pi(x) P(x, y)=\pi(y) P(y, x)$ then $\pi$ is the limiting distribution for $X_{n}$ $\pi(x) P(x, y)=\pi(y) P(y, x)$ is called the detailed balanced equation. In short, the algorithm models a random walk but between each step it adapts the next random step so that the detailed balanced equation is verified. Asymptotically, the MC behaves like the stationary distribution and using Monte Carlo method one can compute the distribution after convergence of the Markov Chain. In our case the state space has 6 or 7 dimensions and the Markov Chain we aim to build is $X_{n}=\left(\gamma_{s m p l, n}, h_{e, n}, \omega_{n}\right)$ and since we are interested in the posterior distribution, in our case we choose:

$$
\begin{aligned}
& \pi\left(\gamma, h_{e}, \omega\right)=\frac{P\left(T_{c w, \text { meas }}, q_{c w, \text { meas }}, T_{w, \text { meas }}, \varepsilon_{\text {meas }}, P_{\mathrm{d}, \text { meas }}, P_{\mathrm{s}, \text { meas }} \mid \gamma, \omega, h_{e}\right)}{P\left(T_{c w, \text { meas }}, q_{c w, \text { meas }}, T_{w, \text { meas }}, \varepsilon_{\text {meas }}, P_{\mathrm{d}}, P_{\mathrm{s}}\right)} \\
& \times P\left(\gamma, \omega, h_{e}\right),
\end{aligned}
$$

that can be computed up to a normalization factor. The advantage of the Metropolis Hasting (MH) algorithm is that it only uses the ratio

$$
R=\frac{\pi\left(\gamma_{n}, h_{e, n}, \omega_{n}\right) P\left(\gamma_{n}, h_{e, n}, \omega_{n} \mid \gamma_{n-1}, h_{e, n-1}, \omega_{n-1}\right)}{\pi\left(\gamma_{n-1}, h_{e, n-1}, \omega_{n-1}\right) P\left(\gamma_{n-1}, h_{e, n-1}, \omega_{n-1} \mid \gamma_{n}, h_{e, n}, \omega_{n}\right)} .
$$

Since the priors for $h_{e, n}, \omega_{n}$ are uniform and $\gamma$ follows a beta distribution, the ratio simplifies into:

$$
\begin{aligned}
R= & \frac{P\left(T_{c w, \text { meas }}, q_{c w, \text { meas }}, T_{w, \text { meas }}, \varepsilon_{\text {meas }}, P_{\mathrm{d}, \text { meas }}, P_{\mathrm{s}, \text { meas }} \mid \gamma_{n}, h_{e, n} \omega_{n}\right)}{P\left(T_{c w, \text { meas }}, q_{c w, \text { meas }}, T_{w, \text { meas }}, \varepsilon_{\text {meas }}, P_{\mathrm{d}, \text { meas }}, P_{\mathrm{s}, \mathrm{i}} \mid \gamma_{s m p l, n}, h_{e, n} \omega_{n}\right)} \\
& \times \frac{\left(\gamma_{n}-\gamma_{\max }\right)^{2}\left(\gamma_{n}-\gamma_{\text {min }}\right)^{2} P(n-1 \rightarrow n)}{\left(\gamma_{n-1}-\gamma_{\max }\right)^{2}\left(\gamma_{n-1}-\gamma_{\min }\right)^{2} P(n \rightarrow n-1)},
\end{aligned}
$$

where $P(n-1 \rightarrow n)$ is the probability to go from state $n-1$ to $n$. If the random walk is symmetrical $P(n-1 \rightarrow n)=P(n \rightarrow n-1)$ and the ratio is 
1. In our case for an efficient exploration of the distribution of $\gamma$ it is natural to choose the random walk as

$$
\begin{gathered}
h_{e, n}=h_{e, n-1}+\xi_{1} \\
\omega_{n}=\omega_{n-1}+\xi_{2} \\
\log \left(\gamma_{n}\right)=\log \left(\gamma_{n-1}\right)+\xi_{3}
\end{gathered}
$$

Therefore the random walk is not symmetrical for $\gamma$ and the ratio becomes:

$$
\begin{aligned}
R= & \frac{P\left(T_{c w, \text { meas }}, q_{c w, \text { meas }}, T_{w, \text { meas }}, \varepsilon_{\text {meas }}, P_{\mathrm{d}, \text { meas }}, P_{\mathrm{s}, \text { meas }} \mid \gamma_{n}, h_{e, n} \omega_{n}\right)}{P\left(T_{c w, \text { meas }}, q_{c w, \text { meas }}, T_{w, \text { meas }}, \varepsilon_{\text {meas }}, P_{\mathrm{d}, \text { meas }}, p_{\mathrm{s}, \text { meas }} \mid \gamma_{n}, h_{e, n} \omega_{n}\right)} \\
& \times \frac{\left(\gamma_{n}-\gamma_{\max }\right)^{2}\left(\gamma_{n}-\gamma_{\text {min }}\right)^{2} \gamma_{n-1}}{\left(\gamma_{n-1}-\gamma_{\max }\right)^{2}\left(\gamma_{n-1}-\gamma_{\text {min }}\right)^{2} \gamma_{n}}
\end{aligned}
$$

The rest of the algorithm of the implementation follows the $\mathrm{MH}$ algorithm described in [42].

\section{References}

[1] O. Chazot, F. Panerai, in: E. Josyula (Ed.), Hypersonic Nonequilibrium Flows: Fundamentals and Recent Advances, Progress in Astronautics and Aeronautics, American Institute of Aeronautics and Astronautics, Inc., Reston, VA, 2015, pp. 329-342.

[2] A. F. Kolesnikov, in: RTO-EN-AVT-008 - Measurement Techniques for High Enthalpy and Plasma Flows.

[3] J. Marschall, D. A. Pejakovic, W. G. Fahrenholtz, G. E. Hilmas, S. Zhu, J. Ridge, D. G. Fletcher, C. O. Asma, J. Thömel, Journal of Thermophysics and Heat Transfer 23 (2009) 267-278. 
[4] F. Panerai, O. Chazot, Materials Chemistry and Physics 134 (2012) $597-607$.

[5] J. Marschall, D. Pejakovic, W. G. Fahrenholtz, G. E. Hilmas, F. Panerai, O. Chazot, Journal of Thermophysics and Heat Transfer 26 (2012) 559572.

[6] G. Herdrich, M. Fertig, D. Petkow, A. Steinbeck, S. Fasoulas, Progress in Aerospace Sciences 48âĂŞ49 (2012) 27 - 41. Assessment of Aerothermodynamic Flight Prediction ToolsAssessment of Aerothermodynamic Flight Prediction Tools.

[7] F. Panerai, B. Helber, O. Chazot, M. Balat-Pichelin, Carbon 71 (2014) $102-119$.

[8] M. Balat-Pichelin, L. Charpentier, F. Panerai, O. Chazot, B. Helber, K. Nickel, Journal of the European Ceramic Society 35 (2015) 487-502.

[9] B. Massuti-Ballester, S. Pidan, G. Herdrich, M. Fertig, Advances in Space Research 56 (2015) $742-765$.

[10] D. Fletcher, J. Meyers, Journal of Thermophysics and Heat Transfer (2016) 1-11.

[11] S. A. Vasil'evskii, A. N. Gordeev, A. F. Kolesnikov, Fluid Dynamics 52 (2017) 158-164.

[12] B. Helber, C. O. Asma, Y. Babou, A. Hubin, O. Chazot, T. E. Magin, Journal of Materials Science 49 (2014) 4530-4543. 
[13] T. M. Cheung, F. F. Schrijer, G. Park, Journal of Spacecraft and Rockets (2016) 644-653.

[14] R. Upadhyay, K. Miki, O. Ezekoye, J. Marschall, Experimental Thermal and Fluid Science 35 (2011) 1588 - 1599.

[15] A. Turchi, P. M. Congedo, T. E. Magin, International Journal of Thermal Sciences (2017) - .

[16] F. Sanson, N. Villedieu, F. Panerai, O. Chazot, P. M. Congedo, T. E. Magin, Experimental Thermal and Fluid Science 82 (2017) 414 - 423.

[17] S. Cheung, K. Miki, E. Prudencio, C. Simmons, Chemical Physics 475 (2016) 136-152. Cited By 1.

[18] G. Terejanu, R. R. Upadhyay, K. Miki, Experimental Thermal and Fluid Science 36 (2012) 178 - 193.

[19] A. F. Kolesnikov, Fluid Dynamics 28(1) (1993) 131-137.

[20] J. Greaves, J. Linnett, Transactions of the Faraday Society 54 (1958) $1323-1330$.

[21] R. A. Young, The Journal of Chemical Physics 34 (1961) 1295-1301.

[22] G. M. Prok, Effect of surface preparation and gas flow on nitrogen atom surface recombination, Technical Report NASA TN-D-1090, NASA Lewis Research Center, Cleveland, OH, United States, 1961.

[23] D. E. Rosner, AIAA Journal 2 (1964) 945-948. 
[24] H. H. Ammann, Heterogeneous recombination and heat transfer with dissociated nitrogen, Ph.D. thesis, Purdue University, 1964.

[25] P. Dickens, M. Sutcliffe, Transactions of the Faraday Society 60 (1964) $1272-1285$.

[26] R. Hartunian, W. Thompson, S. Safron, The Journal of Chemical Physics 43 (1965) 4003-4006.

[27] J. May, J. Linnett, Journal of Catalysis 7 (1967) 324-341.

[28] R. B. Pope, AiAA journal 6 (1968) 619-626.

[29] G. A. Melin, R. Madix, Transactions of the Faraday Society 67 (1971) $198-211$.

[30] L. Anderson, AIAA Journal 11 (1973) 649-656.

[31] P. Cauquot, S. C. Cavadias, J. Amouroux, Journal of thermophysics and heat transfer 12 (1998) 206-213.

[32] C. Park, G. A. Raiche, D. M. Driver, J. Olejniczak, I. Terrazas-Salinas, T. M. Hightower, T. Sakai, Journal of thermophysics and heat transfer 20 (2006) 672-679.

[33] G. Park, Journal of Spacecraft and Rockets 50 (2013) 540-555.

[34] A. Nawaz, D. M. Driver, I. Terrazas-Salinas, S. A. Sepka, in: 44th AIAA Thermophysics Conference, AIAA 2013-3138.

[35] A. Cipullo, B. Helber, F. Panerai, O. Chazot, Journal of Thermophysics and Heat Transfer, AIAA 28 (2014) 381-393. 
[36] A. Viladegut, O. Chazot, in: 45th AIAA Thermophysics Conference, p. 3107.

[37] D. M. Driver, S. A. Sepka, in: 45th AIAA Thermophysics Conference, AIAA 2015-2666, American Institute of Aeronautics and Astronautics, 2011.

[38] P. F. Barbante, Accurate and Efficient Modelling of High Temperature Nonequilibrium Air Flows, Ph.D. thesis, Université Libre de Bruxelles, von Karman Institute for Fluid Dynamics, 2001.

[39] P. F. Barbante, O. Chazot, Journal of Thermophysics and Heat Transfer 20 (2006) 493-499.

[40] B. Bottin, D. P. Vanden Abeele, M. Carbonaro, G. Degrez, G. S. R. Sarma, Journal of Thermophysics and Heat Transfer 13 (1999) 343-350.

[41] M. Plummer, Statistics and Computing 25 (2014) 37-43.

[42] J. Kaipio, E. Somersalo, Statistical and Computational Inverse Problems, volume 160 of Applied Mathematical Sciences, Springer-Verlag, New York, 2005.

[43] A. Gelman, J. Carlin, H. Stern, D. Dunson, A. Vehtari, D. Rubin, Bayesian Data Analysis, Third Edition, Chapman \& Hall/CRC Texts in Statistical Science, Taylor \& Francis, 2013.

[44] R. E. Bellman, S. E. Dreyfus, Applied dynamic programming, Princeton university press, 2015. 
[45] J. Foo, G. E. Karniadakis, Journal of Computational Physics 229 (2010) $1536-1557$.

[46] H. Haario, E. Saksman, J. Tamminen, Bernoulli 7 (2001) 223-242.

[47] C. Andrieu, J. Thoms, Statistics and Computing 18 (2008) 343-373.

[48] P. Bremaud, Markov Chains: Gibbs Fields, Monte Carlo Simulation, and Queues, Texts in Applied Mathematics, Springer New York, 2001.

[49] P. Diaconis, Bulletin of the American Mathematical Society 46 (2009) 179-205.

[50] N. Metropolis, A. W. Rosenbluth, M. N. Rosenbluth, A. H. Teller, E. Teller, The Journal of Chemical Physics 21 (1953) 1087. 